\title{
О ПОТЕРЕ УСТОЙЧИВОСТИ СЖАТОГО СТЕРЖНЯ ПРИ РАСПРЕДЕЛЁННЫХ НАГРУЗКАХ ОТ СОБСТВЕННОГО ВЕСА
}

\author{
Аккулова Мадина Муслимовна \\ студент, лаборант
}

Научный руководитель: Культербаев Хусен Пшимурзович

д.т.н., профессор

СКФУ, Северо-Кавказский центр математических исследований

Аннотация: Рассматривается неклассическая задача о потере устойчивости прямолинейного вертикального стержня, нагруженного равномерно распределённой нагрузкой. Нижний конец стержня жёстко защемлён, верхний свободен от нагрузок. Математическая модель задачи состоит из основного дифференциального уравнения изогнутой оси стержня, дополненного граничными условиями. Они с помощью метода конечных разностей приводятся к однородной системе алгебраических уравнений с квадратной матрицей. Рассмотрены стержни с постоянным сечением по длине. Критические плотности распределённой нагрузки как неизвестные величины содержатся в системе уравнений, из которой извлекаются численнографическим способом с помощью вычислительного комплекса Matlab. Сделаны выводы, имеющие практическое значение.

Ключевые слова: Математическая модель; критическая нагрузка; дифференциальные уравнения продольного изгиба; граничные условия; система алгебраических уравнений; метод конечных разностей.

\section{ABOUT THE LOSS OF STABILITY OF THE COMPRESSED ROD WITH DISTRIBUTED LOADS FROM ITS WEIGHT}

\section{Akkulova Madina Muslimovna Kulterbaev Khusen Pshimurzovich}

\begin{abstract}
A non-classical problem of the loss of stability of a straight vertical bar loaded with a uniformly distributed load is considered. The lower end of the rod is rigidly clamped, the upper end is free from loads. The mathematical model of the


problem consists of the basic differential equation of the curved axis of the bar, supplemented by boundary conditions. They are reduced using the finite difference method to a homogeneous system of algebraic equations with a square matrix. Bars with a constant cross-section along the length are considered. Critical densities of the distributed load as unknown quantities are contained in the system of equations, from which they are extracted numerically and graphically using the Matlab computer complex. Conclusions of practical importance are drawn.

Key words: Mathematical model; critical load; differential equations of buckling; border conditions; system of algebraic equations; finite difference method.

\section{1. Введение}

Длинные вертикальные стержневые конструкции, обладающие значительным собственным весом, широко распространены в практике возведения опор линий передач, прожекторов, антенн, мачт, ветроэнергетических установок и т. д. Их столь широкое распространение стимулирует постоянный интерес к вопросам их устойчивости от распределенной нагрузки собственного веса. В таких сооружениях продольные сжимающие силы непрерывно распределены вдоль стержня. Поэтому дифференциальное уравнение кривой прогибов не является простым уравнением с постоянными коэффициентами и его решение существенно усложняется.

При значительной величине распределённой нагрузки стержень теряет прямолинейное вертикальное положение и происходит переход к криволинейному положению равновесия (рис. 1).

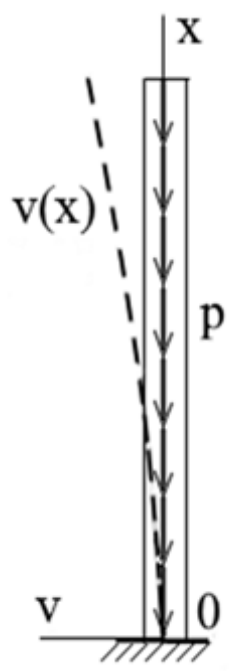

Рис. 1. 
В данной работе ставится задача об определении критической

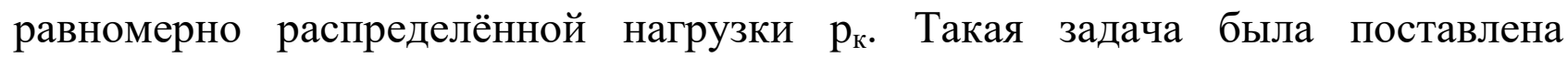
Эйлером и рассматривалась отечественными и зарубежными авторами [1-4].

\section{2. Математическое моделирование задачи}

В отклоненном положении основное дифференциальное уравнение имеет вид.

$$
\mathrm{bv}^{\prime \prime \prime}+\mathrm{pxv}^{\prime}=0, \quad \mathrm{x} \in(0 ; l), \quad \mathrm{b}=\mathrm{EJ} .
$$

Здесь штрих в верхнем индексе соответствует дифференцированию по аргументу $\mathrm{x}, \mathrm{p}$ - удельный вес стержня, отнесенная к единице длины стержня. Далее это уравнение дополняется граничными условиями. Решение такой задачи аналитическими методами представляет значительные сложности и проводится с помощью сложных бесселевых функций.

В данной работе предлагается более удобная математическая модель задачи, которая реализуется численным методом конечных разностей [5-9] с помощью компьютерных технологий.

По расчетной схеме стержня, представленной на рис. 1 определяются четыре очевидные краевые условия

$$
\mathrm{v}(0)=0, \quad \mathrm{v}^{\prime}(0)=0, \quad \mathrm{M}(l)=0, \quad \mathrm{Q}(l)=0,
$$

где $\mathrm{M}$ и $\mathrm{Q}$ - изгибающие моменты и поперечные силы в сечениях стержня. Первые два условия на нижнем конце кинематические и вытекают из защемления нижнего конца. Здесь перемещение $\mathrm{v}$ и угол поворота $\mathrm{v}^{\prime}$ невозможны. Третье и четвертое условия соответствуют свободному верхнему концу и являются статическими. Здесь какие-либо силовые воздействия отсутствуют, поэтому изгибающий момент и поперечная сила в сечении равны нулю. В совокупности уравнения (1), (2) образуют математическую модель задачи.

В силу соображений, высказанных выше, перейдем от континуальной области рассмотрения задачи к дискретной сеточной области. Заменим в (1) (2) область непрерывного изменения аргумента $\mathrm{x}$ областью дискретного изменения (рис. 2)

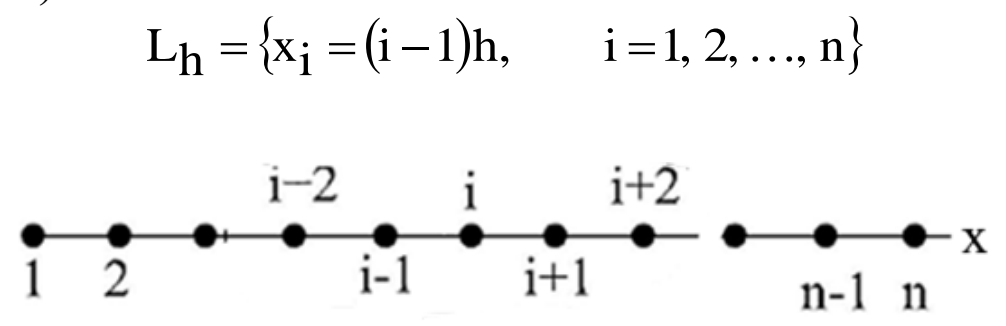

Рис. 2. 
с шагом $\mathrm{h}=l /(\mathrm{n}-1)$. Вместо непрерывной функции $\mathrm{v}(\mathrm{x})$ будем рассматривать сеточную функцию $\mathrm{v}_{\mathrm{i}} \approx \mathrm{v}\left(\mathrm{x}_{\mathrm{i}}\right), \mathrm{i}=1,2, \ldots, \mathrm{n}$. Далее воспользуемся численным методом конечных разностей $[\ldots]$ и представим прогибы $\mathrm{v}(\mathrm{x})$ и их производные в совокупном виде

$$
\begin{gathered}
\mathrm{v}=\mathrm{v}_{\mathrm{i}}, \quad \mathrm{v}_{\mathrm{i}}^{\prime}=\frac{1}{2 \mathrm{~h}}\left(-\mathrm{v}_{\mathrm{i}-1}+\mathrm{v}_{\mathrm{i}+1}\right), \quad \mathrm{v}_{\mathrm{i}}^{\prime \prime}=\frac{1}{\mathrm{~h}^{2}}\left(\mathrm{v}_{\mathrm{i}-1}-2 \mathrm{v}_{\mathrm{i}}+\mathrm{v}_{\mathrm{i}+1}\right), \\
\mathrm{v}^{\prime \prime \prime}=\frac{1}{2 \mathrm{~h}^{3}}\left(-\mathrm{v}_{\mathrm{i}-2}+2 \mathrm{v}_{\mathrm{i}-1}-2 \mathrm{v}_{\mathrm{i}+1}+\mathrm{v}_{\mathrm{i}+2}\right), \\
\mathrm{v}^{\prime \prime \prime \prime}=\frac{1}{\mathrm{~h}^{4}}\left(\mathrm{v}_{\mathrm{i}-2}-4 \mathrm{v}_{\mathrm{i}-1}+6 \mathrm{v}_{\mathrm{i}}-4 \mathrm{v}_{\mathrm{i}+1}+\mathrm{v}_{\mathrm{i}+2}\right) .
\end{gathered}
$$

С их помощью переведём постановку задачи (1), (2) в конечноразностную форму.

Выражения для изгибающего момента $\mathrm{M}$ и поперечной силы Q в (2) необходимо превратить в уравнения. С этой целью представим их с помощью следующих известных формул механики конструкций

$$
\mathrm{Q}(\mathrm{x})=\mathrm{bv}^{\prime \prime \prime}(\mathrm{x}), \quad \mathrm{M}(\mathrm{x})=\mathrm{bv}^{\prime \prime}(\mathrm{x}) \text {. }
$$

$\mathrm{C}$ помощью метода конечных разностей и выражений (3) обратим математическую модель (1) - (2) в систему алгебраических уравнений с помощью производных (3).

Пропуская тривиальные преобразования, можно основное уравнение (1) представить в методе конечных разностей в виде

$$
\begin{aligned}
-\mathrm{v}_{i-2}+\delta \mathrm{v}_{\mathrm{i}-1}+\varepsilon \mathrm{v}_{\mathrm{i}+1}+\mathrm{v}_{\mathrm{i}+2}=0, \quad i=3,4, \ldots, n-2, \\
\delta=2-\mathrm{cx}_{\mathrm{i}} \mathrm{h}^{2}, \quad \mathrm{c}=\mathrm{p} / \mathrm{b}, \quad \varepsilon=-2+\mathrm{cx}_{\mathrm{i}} \mathrm{h}^{2} .
\end{aligned}
$$

К нему добавляются краевые условия. На нижнем конце:

$$
\mathrm{v}_{1}=0, \quad-3 \mathrm{v}_{1}+4 \mathrm{v}_{2}-\mathrm{v}_{3}=0 \text {. }
$$

На верхнем конце:

$$
\begin{aligned}
& 3 v_{n-4}-14 v_{n-3}+24 v_{n-2}-18 v_{n-1}+5 v_{n}=0, \\
& -v_{n-3}+4 v_{n-2}-5 v_{n-1}+2 v_{n}=0 .
\end{aligned}
$$

Уравнения (4) - (7) образуют алгебраическую систему, которую запишем в матрично-векторном виде

$$
\mathrm{Bv}=0 \text {, }
$$

где В - квадратная матрица порядка $\mathrm{n}, \mathrm{v}$ - вектор-столбец с компонентами $\mathrm{v}_{\mathrm{i}}, \mathrm{i}=1,2, \ldots, \mathrm{n}$. Уравнение (8) имеет очевидное решение $\mathrm{v}=(0,0, \ldots, 0)^{\mathrm{T}}$, которое не представляет интереса. Ненулевое решение возможно при нулевом значении определителя матрицы В.

$$
\operatorname{det}[B(p)] v=0 \text {, }
$$


где В - квадратная матрица порядка n, v - вектор-столбец. В развёрнутом виде уравнение (9) имеет вид

$$
\left(\begin{array}{ccccccccc}
1 & & & & & & & & \\
-3 & 4 & -1 & & & & & & \\
-1 & \delta & 0 & \varepsilon & 1 & & & & \\
\cdots & \cdots & \cdots & \cdots & \cdots & \ldots & \cdots & \ldots & \cdots \\
& & & & -1 & \delta & 0 & \varepsilon & 1 \\
& & & & 3 & -14 & 24 & -18 & 5 \\
& & & & & -1 & 4 & -5 & 2
\end{array}\right)\left(\begin{array}{c}
v_{1} \\
v_{2} \\
v_{3} \\
\cdots \\
v_{n-2} \\
v_{n-1} \\
v_{n}
\end{array}\right)=\left(\begin{array}{c}
0 \\
0 \\
0 \\
\cdots \\
0 \\
0 \\
0
\end{array}\right),
$$

где нулевые элементы не показаны. Ненулевые значения вектора v возможны лишь при нулевом значении определителя матрицы В

$\operatorname{det}[(\mathrm{B}(\mathrm{p})]=0$.

Значения р, при которых выполняется уравнение (10) и являются критическими распределёнными нагрузками сжатого стержня. Определить эти значения при больших значениях $\mathrm{n}$ простыми способами невозможно. Разумный выход из такого затруднения состоит в использовании численно-графического метода определения критических сил [11-12]. Его суть в том, что метод конечных разностей применяется в сочетании с использованием компьютерных программ и вычислительных комплексов. По результатам вычислений строится график $\mathrm{p}-\operatorname{det}(\mathrm{B})$. Те значения $\mathrm{p}$, при которых определитель равен нулю, с большой точностью считываются с экрана монитора при визуальном рассмотрении графиков. Они и есть критические

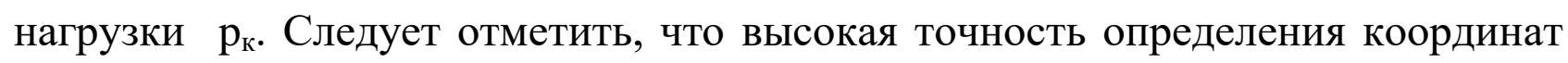
точек на оси р обеспечивается наличием у вычислительных комплексов «лупы», позволяющей многократно увеличивать фрагменты рисунков.

Перейдём к рассмотрению конкретного примера/

Пример. Для стального стержня круглого поперечного сечения на рис. 1 даны параметры: $l=3 \mathrm{M}, \mathrm{E}=2,1 \cdot 10^{11} \Pi \mathrm{a}, \mathrm{d}=2,5 \mathrm{~cm}$.

Требуется найти первые критические значения распределённой нагрузки.

В системе вычислительного комплекса Matlab была составлена компьютерная программа и получен график, представленный на рис. 3. По нему прочитаны первые три значения плотности распределённой нагрузки

$$
\mathrm{p}_{\kappa}=\{0,5191 ; 6,5901 ; 19,2888\} \kappa \mathrm{\kappa} / \mathrm{M} \text {. }
$$




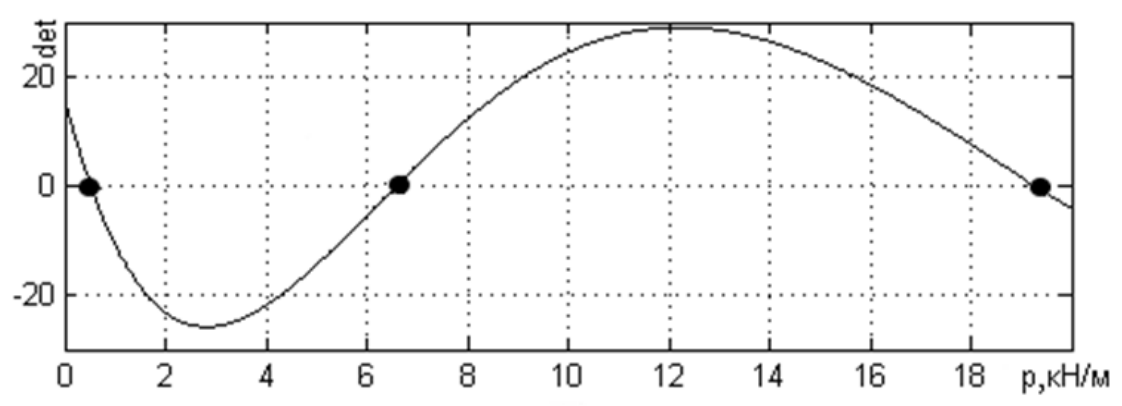

Рис. 3.

Значения критических сил на рисунке отмечены жирными точками.

Из приведённых критических нагрузок только первая представляет практический интерес. Остальные нагрузки имеют лишь теоретическое значение. Собственные формы потери устойчивости, соответствующие критическим нагрузкам, могут оказаться полезными в динамических задачах.

Следует отметить, что определение критических нагрузок с помощью численно-графического метода не представило каких-либо сложностей.

\section{3. Выводы}

1. Решение задач устойчивости стержней при распределённых нагрузках численно-графическим методом имеет преимущества по сравнению с известными аналитическими методами.

2. Сочетание метода конечных разностей и вычислительного комплекса Matlab является эффективным инструментом при решении задач устойчивости стержней.

\section{Список литературы}

1. Феодосьев В.И. Сопротивление материалов. -М.: Изд. МГТУ им. Н.Э. Баумана, 2003. - 592 с.

2. Вольмир А.С. Устойчивость деформируемых систем. М.: Наука. 1967. $-984 \mathrm{c}$.

3. Прочность, устойчивость, колебания. Справочник в трёх томах. Том 3. Под редакцией Биргера И.А., Пановко Я.Г. М.: Машиностроение. 1968. - 567 с.

4. Строительная механика. Динамика и устойчивость сооружений. М.: Наука, Стройиздат, 1984. - 416 с.

5. Самарский А.А., Гулин А.В. Численные методы. М.: Наука, Гл. ред. физ.-мат. лит.,1989. - 432 с. 
6. Варвак П.М., Варвак Л.П. Метод сеток в задачах расчёта строительных конструкций. М.: Стройиздат, 1977. - 154 с.

7. Вержбицкий В.М. Основы численных методов. М.: Высшая школа, 2002. $-840 \mathrm{c}$.

8. Ильин В.П., Карпов В.В., Масленников А.М. Численные методы решения задач строительной механики. - М.: Изд-во АСВ; СПб.: СПбГАСУ, 2005. - 425 c.

9. Караманский Т.Д. Численные методы строительной механики. -М.: Стройиздат, 1981. - 436 с.

10. Культербаев Х.П., Барагунова Л.А. О реализации проблемы собственных значений сжато-растянутого стержня на компьютере. Компьютерные технологии в строительстве: Материалы Всероссийской научно-технической конференции. ДГТУ. - Махачкала: Алеф (ИП Овчинников), 2012. С. 90-94.

11. Kulterbaev Kh.P., Baragunova L.A., Shogenova M.M., Senov Kh.M. About a High-Precision Graphoanalytical Method of Determination of Critical Forces of an Oblate Rod. Proceedings 2018 IEEE International Conference "Quality Management, Transport and Information Security, Information Technologies" (IT\&QM\&IS). September, 24-28, 2018. St. Petersburg . Russia 2018. P. 794-796. 